\title{
La Situation Des Enfants En Dehors Du Systeme Scolaire En Côte d'Ivoire
}

\author{
Dr. Sika Glebelho Lazare \\ Enseignant-chercheur à l'Ecole Nationale Supérieure de Statistique et \\ d'Economie Appliquée, Abidjan, Côte d'Ivoire
}

Kacou Amoin Elise

Doctorante en démographie à Institut de Démographie de l'Université Paris 1/Ecole Nationale Supérieure de Statistique et d'Economie Appliquée, Abidjan, Côte d'Ivoire

\section{Doi:10.19044/esj.2018.v14n31p1 ～URL:http://dx.doi.org/10.19044/esj.2018.v14n31p1}

\begin{abstract}
One of the goals of Education for All (EFA) was to ensure that by 2015 children, specifically girls, vulnerable children and those belonging to ethnic minorities, have access to compulsory and free quality primary education and follow it to completion. However, at the end of 2015, Ivory Coast had not been able to achieve the objectives set, particularly the universal schooling of children and adolescents. The country faces many constraints related to its education system, notably school exclusion. The problem of access and maintenance of children in the system, whether they relate to the demand for or supply of education, remain important. The 2002 political crisis has exacerbated the obstacles to children's schooling because of the massive displacement of populations and the lack of infrastructures. What are the main factors behind school exclusion? how many children and adolescents are out of school? what are the economic implications? To answer these questions, we rely on data from the national survey of out of school children and adolescents conducted in 2015. The study reveals that 1,550,586 children aged 3-5; 1,123,674 children aged 6-11, 797137 of 12-15 years are out of the school system. In order to achieve economic emergence, the country must imperatively address the problem of access to education with the utmost speed.
\end{abstract}

Keywords: Children, school exclusion, demographic dividend, Ivory Coast.

\section{Résumé}

L'un des objectifs de l'Education Pour Tous (EPT) était de faire en sorte qu'en 2015 tous les enfants, notamment les filles, les enfants en difficulté et ceux appartenant à des minorités ethniques, aient la possibilité d'accéder à 
un enseignement primaire obligatoire et gratuit de qualité et de le suivre jusqu'à son terme. Cependant, à la fin de cette échéance, la Côte d'Ivoire n'a pu atteindre les objectifs fixés, notamment la scolarisation universelle. De plus, le pays est confronté à de nombreuses contraintes liées au système éducatif, surtout la question des enfants et adolescents en dehors de l'école. Les problèmes d'accès et de maintien des enfants dans le système qu'ils soient relatifs à la demande ou à l'offre d'éducation restent importants. La crise politique de 2002 a exacerbé les entraves à la scolarisation à cause du déplacement massif des populations et à l'insuffisance des structures dans les zones d'accueil. Mais quels sont les principaux facteurs à l'origine de l'exclusion scolaire? Combien d'enfants et d'adolescents sont-ils hors de l'école ? Quelles sont les implications sur le plan économique ? Pour répondre à ces interrogations, nous nous appuyons sur les données issues de l'enquête nationale sur les enfants et adolescents hors du système scolaire réalisée en 2015. Elles révèlent que 1550586 enfants de 3-5 ans, 1123674 enfants de 611 ans, 797137 de 12-15 ans sont hors du système scolaire. Dans la perspective de parvenir à l'émergence économique, le pays doit, de façon impérative, adresser le problème de l'éducation des enfants avec la plus grande célérité.

Mots clés : Enfants, exclusion scolaire, dividende démographique, Côte d'Ivoire.

\section{Introduction}

L'éducation est un droit fondamental nécessaire au développement de l'individu et de la société. De ce fait, tous les enfants du monde doivent en bénéficier. Ce droit est inaliénable, ininterruptible et permanent dans les sociétés, même dans un contexte de crise (Fadiga, 2006). Toutefois, dans de nombreuses régions du monde, notamment dans les pays en développement, des millions d'enfants sont en dehors du système scolaire. Ce phénomène est plus visible en Afrique subsaharienne, en Asie du Sud et de l'Ouest. Les estimations conjointes de l'Institut de Statistique de l'UNESCO (ISU) et le Rapport mondial de suivi sur l'éducation (GEM) font état de 263 millions d'enfants en dehors de l'école. Ce qui représente près d'un quart de la population européenne (UNESCO, 2016). Dans cette population, 61 millions d'enfants sont en âge d'aller à l'école primaire (6-11 ans), 60 millions d'adolescents sont en âge d'être inscrits dans le premier cycle du secondaire (12-15 ans), et 142 millions de jeunes sont en âge d'être inscrits dans le deuxième cycle du secondaire (16-18 ans).

L'Afrique subsaharienne est la région du monde où les taux d'exclusion sont les plus élevés. En effet, plus de $20 \%$ des enfants âgés de 6 à 11 ans ne vont pas à l'école, suivis d'un tiers des jeunes âgés de 12 à 15 ans et près de $60 \%$ des jeunes de 16 à 18 ans ne sont pas scolarisés 
(UNESCO, 2016). Cette population présente les différentes caractéristiques liées notamment au sexe (les filles sont plus touchées que les garçons), à l'âge (le sous-âge et surtout le sur-âge sont positivement associés à l'abandon), au milieu de résidence (les enfants d'âge scolaire primaire vivant dans les zones rurales sont exclus de l'école par rapport à ceux du milieu urbain), au revenu des ménages (les pauvres risquent 3 fois plus de ne pas être scolarisés que les riches), au niveau d'éducation de la mère (les enfants dont la mère n'a reçu aucun niveau d'instruction présentent deux fois plus de risque d'être non scolarisés). Toutefois, le profil des enfants hors du système éducatif est complexe.

En Côte d'Ivoire, le secteur éducatif recevait, en 2016, près de 5\% du PIB en ressources budgétaires, plaçant ainsi le pays parmi les premiers sur le continent africain. (Groupe Banque mondiale, 2017). Déjà en 2003, le rapport national sur les OMD publié avec l'appui du système des Nations-Unies indiquait des prévisions optimistes à l'échéance 2015 au regard du rythme de croissance des indicateurs d'accès et d'efficacité du système éducatif. Les taux nets de scolarisation et d'achèvement se situaient aux alentours de $69 \%$ contre un objectif mondial de $100 \%$. De même, ces quatre dernières années, le pays enregistre une amélioration de la performance scolaire. Avec des taux d'inscription et de réussite ayant sensiblement augmenté. Malgré les efforts consentis par l'Etat au financement de l'éducation, les objectifs de l'Education Pour Tous (EPT) notamment la scolarisation universelle des enfants et adolescents, n'ont pas été atteints. De plus, la question des enfants en dehors du système éducatif semble prendre de l'ampleur. La crise militaropolitique de 2002 qui a provoqué un exode massif de la population, a constitué une entrave majeure à la bonne marche du processus de scolarisation. Elle a été la cause de la déscolarisation de beaucoup d'enfants et du maintien de nombreux autres en dehors des structures éducatives.

Les données des enquêtes ménages montrent que le phénomène de l'exclusion scolaire est persistant en Côte d'Ivoire. En effet, l'enquête par grappes à indicateurs multiples de 2006 estimait à 30\% le taux des enfants de 6 ans n'ayant pas accès au CP1 (Cours Préparatoire 1), celle de 2012 le situe à $34,5 \%$. Fort de ce constat, cet article qui s'inscrit dans une approche descriptive, a pour objectif d'améliorer les connaissances sur la question de l'exclusion scolaire. En d'autres termes, l'analyse se propose d'identifier les causes profondes de ce phénomène, de déterminer son ampleur et de montrer son implication sur le plan économique.

\section{Evolution de la population d'âge scolaire en Côte d'Ivoire}

La Côte d'Ivoire a amorcé sa transition démographique avec une baisse de la fécondité depuis le début des années 1980. L'indice synthétique de fécondité (ISF) est passé respectivement de 7,2 enfants en 1981 à 6,3 enfants 
par femme en 1988 (MEMPD $\left.{ }^{1}, 2006\right)$. Des données plus récentes confirment cette tendance à la baisse mais le niveau reste encore élevé avec 4,6 enfants par femme en 2016. Sur la base des projections faites à partir du RGPH de 2014, l'INS estime la population ivoirienne à 24571044 habitants en 2017. Cette population est extrêmement jeune : $41,5 \%$ des individus ont moins de 15 ans et les jeunes âgés de 15 à 24 ans représentent 19\% de la population totale (INS, 2014). L'évolution rapide de la population générale s'accompagne de façon inéluctable d'une augmentation significative de celle des enfants notamment ceux en âge de scolarisation. Entre 1998 et 2014, la population d'âge scolaire, celle des enfants âgés de 6 à 11 ans, est passée de 2635627 personnes à 3571 909. Le taux moyen annuel de croissance de cette population de 2,6\%, impliquait un accroissement significatif de l'ensemble des équipements alors disponibles pour garder la même qualité des enseignements. Sur la base d'un effectif moyen de 35 élèves par classe, une telle évolution appelle la construction entre ces deux dates de 2133 salles de classes supplémentaires et la formation du nombre d'instituteurs nécessaires pour animer celles-ci si tous les enfants allaient à l'école.

Le Rapport d'Etat sur le Système Educatif National de 2016 (RESEN) prévoit entre 2016 et 2020 des taux moyens annuels de $2,4 \%$ de croissance des effectifs. En se basant sur ces estimations de croissance, la population des enfants de 3 à 5 ans s'établit à 2485990 enfants et ceux de 6 à 11 ans s'élève à 4160307 enfants. Il est vrai qu'au cours de la phase de la transition démographique dans laquelle la Côte d'Ivoire s'inscrit désormais, le rythme de croissance de l'effectif des enfants est moindre que dans les années 1980, mais ce taux de croissance reste conséquent et nécessite de gros efforts d'investissement dans les infrastructures scolaires et la formation des instituteurs et autres encadreurs.

\section{Principales causes de l'exclusion scolaire en Côte d'Ivoire}

On a d'une part, les causes inhérentes au système éducatif lui-même et d'autre part, les causes externes. Le fonctionnement actuel du système éducatif ivoirien prive l'accès à certains enfants et pousse d'autres vers une sortie progressive et définitive de l'école avant l'obtention d'un diplôme, parfois même avant d'acquérir les notions élémentaires de lecture ou de calcul. Au nombre de ces causes, on peut citer l'insuffisance de l'offre scolaire, le redoublement, l'abandon et l'exclusion «définitive». Quant aux causes externes, elles prennent en compte le manque de perspectives qu'offre l'école, les difficultés financières des parents et surtout la non scolarisation des enfants en situation de handicap.

\footnotetext{
${ }^{1}$ Ministère d'Etat, Ministère du Plan et du Développement
} 


\section{II.1. Causes internes au système éducatif}

\section{- L'insuffisance de l'offre scolaire}

L'offre scolaire est insuffisante dans certaines localités notamment dans les quartiers populaires ou périphériques des grandes villes ainsi que dans des villages isolés. En effet, la demande d'éducation y reste forte, compte tenu du rythme d'accroissement rapide de la population scolarisable. Dans ce cas, certains enfants n'entreront jamais à l'école et il n'est pas rare de voir d'autres inscrits à l'école au-delà même de l'âge légal (7 ou 8 ans voire plus). Ce déficit global de l'offre d'éducation traduit l'incapacité d'accueil en termes d'infrastructures et d'enseignants. En outre, l'insuffisance de l'offre d'éducation entraîne un surpeuplement des classes donc à une mauvaise qualité de l'apprentissage avec comme conséquence, une faible probabilité pour les

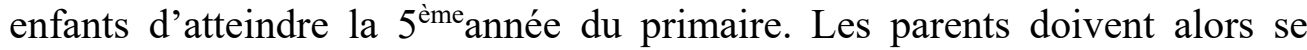
tourner vers les écoles privées pour ceux qui en ont les moyens; à défaut, les enfants ne sont pas scolarisés.

L'insuffisance de l'offre scolaire est particulièrement perceptible au niveau du préscolaire. Comme ce niveau d'enseignement n'est pas obligatoire en Côte d'Ivoire, la participation de l'Etat dans l'offre est particulièrement faible. De plus, les catégories sociales intéressées par ce type d'enseignement étant surtout les populations urbaines ayant des niveaux de vie relativement élevées, on note une certaine concentration des structures de formation préscolaires dans les zones urbaines. Ces structures sont quasiment inexistantes en zones rurales.

\section{- Le redoublement}

La principale cause du redoublement vu de l'intérieur est l'insuffisance des résultats scolaires. Paradoxalement, le redoublement influe négativement sur les résultats scolaires globaux. Il a été établi qu'en moyenne, dans l'enseignement primaire des pays africains, qu'à un point de redoublement supplémentaire correspond une diminution du taux de rétention de 0,8 point et que les pauvres, les ruraux et les jeunes filles sont les premières victimes de redoublement (Mingat et Sosale, 2001). Le redoublement consomme également beaucoup de ressources car il oblige les parents à payer plus d'une fois les mêmes frais. Ce qui est hautement démobilisateurs pour les parents surtout les plus pauvres.

\section{- L'exclusion}

L'exclusion est plus liée à l'âge qu'au résultat scolaire au primaire alors qu'au secondaire, on assiste plutôt au phénomène inverse. Au primaire, la législation scolaire autorise le redoublement une fois par niveau dans les classes intermédiaires jusqu'à ce que l'enfant atteigne l'âge de 15 ans. Au secondaire, l'exclusion est prononcée lorsque les résultats scolaires se situent 
en-dessous du seuil autorisé et que ce seuil peut varier d'un établissement à un autre.

\section{II.2. Causes externes au système éducatif}

\section{- Le manque de perspectives ou d'emplois}

La faible valorisation de l'école est l'une des causes de la non scolarisation ou de l'abandon scolaire des enfants. En effet, lorsque les aînés sortent du système éducatif avec des diplômes et qu'ils ne parviennent pas à obtenir un emploi, les parents démotivés peuvent encourager les plus jeunes à quitter l'école plus tôt pour une mise au travail généralement dans le secteur informel. L'école, dans plusieurs pays africains, ne séduit plus à cause du chômage. Le contenu des cours n'est généralement pas en rapport avec la vie quotidienne des élèves. Du fait de l'inadéquation formation-emploi, de nombreux jeunes diplômés n'arrivent pas à s'insérer sur le marché de l'emploi. Les formations ne sont pas adaptées aux besoins des entreprises. Par exemple, le niveau global du taux combiné du chômage, de la main-d'œuvre potentielle et du sous-emploi est de $27,8 \%$ en 2016 contre $25,3 \%$ en 2014 , soit une hausse de 2,5\% (ENSETE, 2016). Ainsi, le niveau du chômage combiné au sousemploi lié au temps de travail et à la main-d'œuvre potentielle, est plus important chez les femmes $(58,8 \%)$, chez les personnes résidant en milieu urbain (58,2\% dont 27,1\% à Abidjan), chez les jeunes de $14-34$ ans $(73,2 \%)$ et chez les personnes n'ayant aucun niveau d'instruction $(43,1 \%)$.

\section{- La pauvreté}

La pauvreté apparait comme la principale cause des enfants en dehors du système éducatif. Elle explique, en grande partie, la plupart des facteurs d'influence à savoir : le sexe, l'âge, le lieu de résidence, le revenu des ménages, le travail des enfants, le niveau d'instruction de la mère, etc.

En effet, dans les familles aisées, la discrimination du sexe n'est pas perceptible de même que l'inscription tardive qui créée les sur-âges observés surtout dans les classes terminales (CM2 et $\left.3^{\text {ème }}\right)$. En outre, l'impact de la pauvreté sur le phénomène du travail des enfants, le niveau d'instruction de la mère ou le lieu de résidence de la famille a été largement démontré tout au long de l'étude.

\section{- Le problème du handicap}

L'insuffisance et parfois l'inexistence de structures spécialisées pour recevoir les enfants ayant des problèmes particuliers de santé ou en situation de handicap est aussi l'une des raisons qui maintiennent certains enfants en dehors de l'école. Les parents n'ont pas toujours les moyens de s'occuper des enfants présentant par exemple un déficit mental ou nécessitant des soins particuliers. Rares sont, en effet, des structures telles que les instituts 
spécialement dédiés aux malentendants ou aux non-voyants. En Côte d'Ivoire, il existe seulement deux structures publiques s'occupant des enfants en situation de handicap.

\section{Données et méthodes}

L'enquête nationale EHSS menée en 2015 avait pour objectifs principaux d'établir le profil des enfants en dehors de l'école, les raisons fondamentales de ce phénomène et d'identifier les obstacles qui entravent la mise en œuvre des politiques éducatives afin de proposer des stratégies efficientes pour y faire face. Cette enquête a été réalisée sur l'initiative du MENET et de l'UNICEF. Deux approches complémentaires de collecte ont été utilisées : l'une quantitative et l'autre qualitative. Le volet quantitatif a consisté en la collecte d'informations dans des ménages par interview directe, via un questionnaire implémenté sur des tablettes. Après le questionnaire ménage, les enfants, adolescents et jeunes âgés de 3-24 ans jamais scolarisés ou scolarisés, mais ayant abandonné étaient éligibles au questionnaire " enfant " soit une population de 5116 EHSS interviewés. La collecte qualitative s'est faite au travers d'entretiens et de focus groups. Ce volet de l'enquête a ciblé tous les acteurs de l'éducation notamment des élus locaux, des Directeurs Régionaux de l'Education Nationale et de l'Enseignement Technique (DRENET), des leadeurs d'opinion, des responsables de COGES. Les focus groups ont, quant à eux, ciblé les enfants non scolarisés et les enseignants.

\section{Quelles mesures des enfants en dehors de l'école ?}

Le phénomène d'exclusion scolaire des enfants n'est pas simple à cerner et à mesurer. L'ISU et l'UNICEF ont élaboré un cadre conceptuel et une méthodologie qui s'appuie sur cinq dimensions de l'exclusion ${ }^{2}$. En se basant sur l'âge et la situation scolaire, ces cinq dimensions de l'exclusion scolaire sont :

\footnotetext{
${ }^{2}$ Pour les détails de la méthodologie permettant de quantifier l'exclusion scolaire il faut se référer au rapport régional Afrique de l'ouest et du centre de l'UNICEF paru en 2014 « Tous les enfants à l'école d'ici 2015. Initiative mondiale en faveur des enfants non scolarisés ».
} 
- La proportion d'enfants exclus du préscolaire (DE1) c'est-à-dire les enfants âgés de 3 à 5 ans qui ne sont actuellement inscrits ni au préscolaire ni au primaire ;

- La dimension 2 (DE2) fait référence aux enfants exclus du primaire, c'est-à-dire les enfants ayant l'âge d'aller au primaire (6-11 ans), mais qui ne sont inscrits ni au primaire ni au secondaire ;

- La dimension 3 (DE3) concerne les enfants exclus du 1er cycle du secondaire, c'est-à-dire les enfants ayant l'âge d'aller au 1er cycle du secondaire (12-15 ans), mais qui ne sont inscrits ni au primaire ni au secondaire ;

- La dimension 4 (DE4), ce sont les enfants risquant l'exclusion au primaire, c'est-à-dire les enfants encore scolarisés au primaire, peu importe l'âge, mais fortement exposés au risque d'exclusion ;

- La dimension 5 (DE5), les enfants risquant l'exclusion au 1er cycle du secondaire, c'est-à-dire les enfants encore scolarisés au ler cycle du secondaire, peu importe l'âge, mais fortement exposés au risque d'exclusion.

Source : UNICEF/ISU, 2011

\section{IV.1. Profils des enfants en dehors du système scolaire}

L'analyse de l'exclusion scolaire faite sur la base de l'enquête de 2015 révèle l'importance numérique de ce phénomène. Un total de 6061161 enfants de 3-24 ans sont en dehors du système scolaire. Quand on sait que l'éducation favorise le développement humain, le progrès technique et la croissance économique et sociale, comment le pays peut-il espérer atteindre l'émergence économique avec un aussi grand nombre d'enfants non scolarisés ?

Cette exclusion touche davantage les filles ( 3289 391) que les garçons ( 2771 770) mais aussi le milieu rural $(\mathbf{3} 845$ 805) par rapport au milieu urbain ( 2215 356). Les écarts entre filles et garçons pourraient s'expliquer par la scolarisation différentielle selon le genre à l'avantage des garçons et une espérance de vie scolaire plus courte pour les filles. En effet, la conception traditionnelle des rôles contribue finalement à influencer l'investissement de la famille et de la communauté dans l'éducation des enfants. Ainsi, lorsque les ressources sont limitées, les familles opteront pour la scolarisation du garçon au détriment de la fille au motif que le retour sur investissement est plus sûr. Par ailleurs, les enfants non scolarisés sont plus nombreux dans certaines régions du pays notamment au Sud-Ouest, à Abidjan, au Nord et au CentreOuest. Ce constat est probablement lié aux poids des activités économiques dans ces localités. 
Graphique 1 : Répartition par région des enfants et adolescents en dehors de l'école

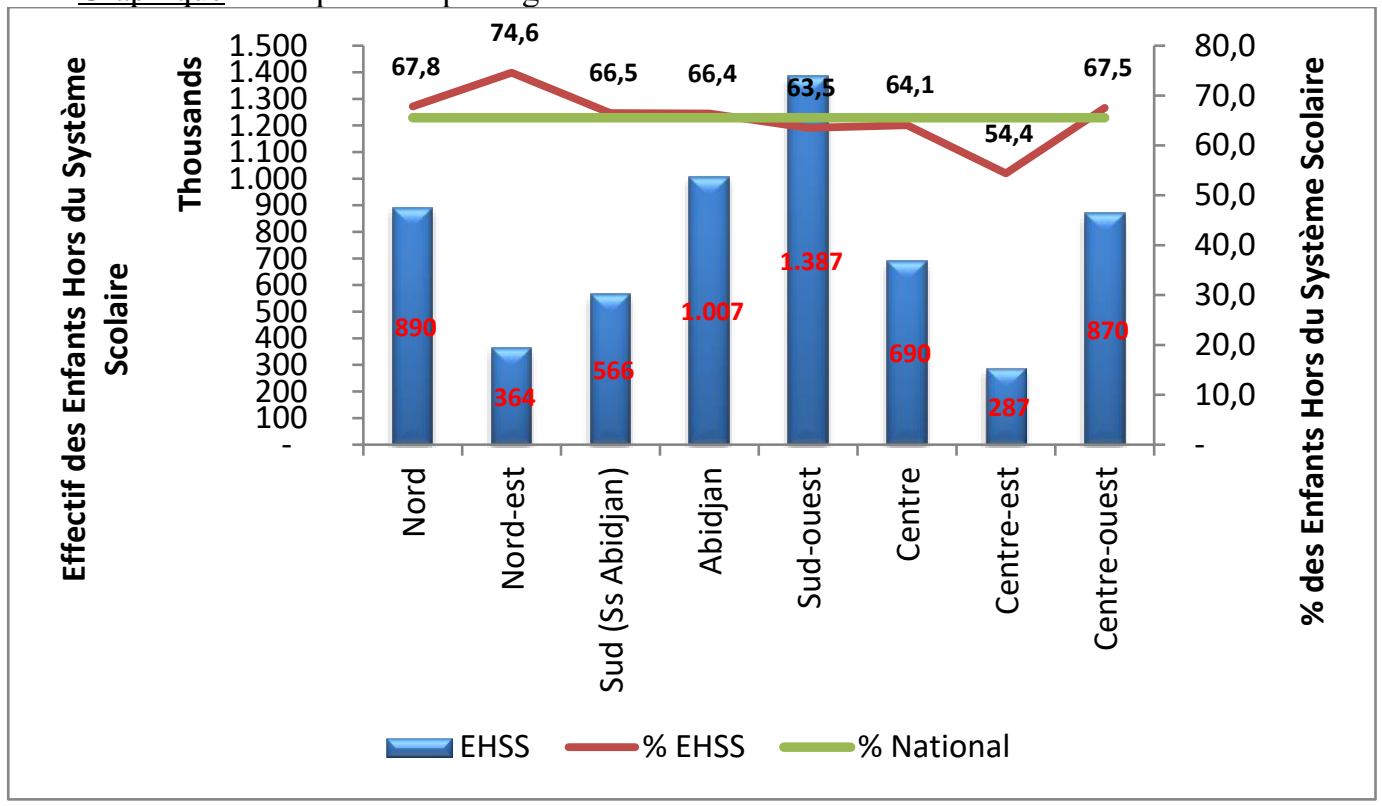

\section{IV.2. Estimations suivant le modèle des 5 dimensions de l'ISU}

En Côte d'Ivoire, les enfants de 3-5 ans sont quasiment hors de l'école (DE1) puisqu'à cet âge, l'admission au préscolaire n'est pas obligatoire. C'est principalement en ville et surtout dans le secteur privé que l'offre préscolaire est développée. La scolarisation au préscolaire est généralement plus courante dans les ménages de niveau de vie élevé. En d'autres termes, le phénomène d'exclusion des enfants au niveau préscolaire est plus intense chez les ménages ayant un niveau de vie plus faible que la moyenne.

En outre, près d'un million d'enfants de 6-11 ans censés être au primaire ne sont pas scolarisés (DE2). Dans cet effectif, on trouve les enfants qui n'ont jamais été à l'école et ceux qui y ont été, mais ont déjà abandonné. Les régions du Nord et du Sud-Ouest ayant connu de fortes perturbations pendant les périodes de crises de 2002 à 2010 sont les plus impactées $(C f$. la carte).

La population des enfants de $12-15$ ans hors du système scolaire s'élève à 797137 (DE3). Elle est deux fois plus importante en milieu rural (533 427) qu'en milieu urbain (263 710). L'implication des adolescents dans les travaux champêtres ainsi que l'absence de collèges dans les territoires ruraux accentuent l'exclusion dans cette tranche d'âge.

La dimension 4 (DE4) rend compte du niveau des risques d'exclusion encouru dans l'enseignement primaire. C'est le cas de certains enfants scolarisés mais n'ayant pas encore accédé au cycle secondaire et qui ont un risque de ne jamais atteindre ce dernier. Leur nombre est estimé à près de 706000 enfants. 
Le risque d'exclusion n'est pas négligeable pour les enfants au premier cycle du secondaire (DE5). Il est toutefois moins important que celui observé au niveau du primaire.

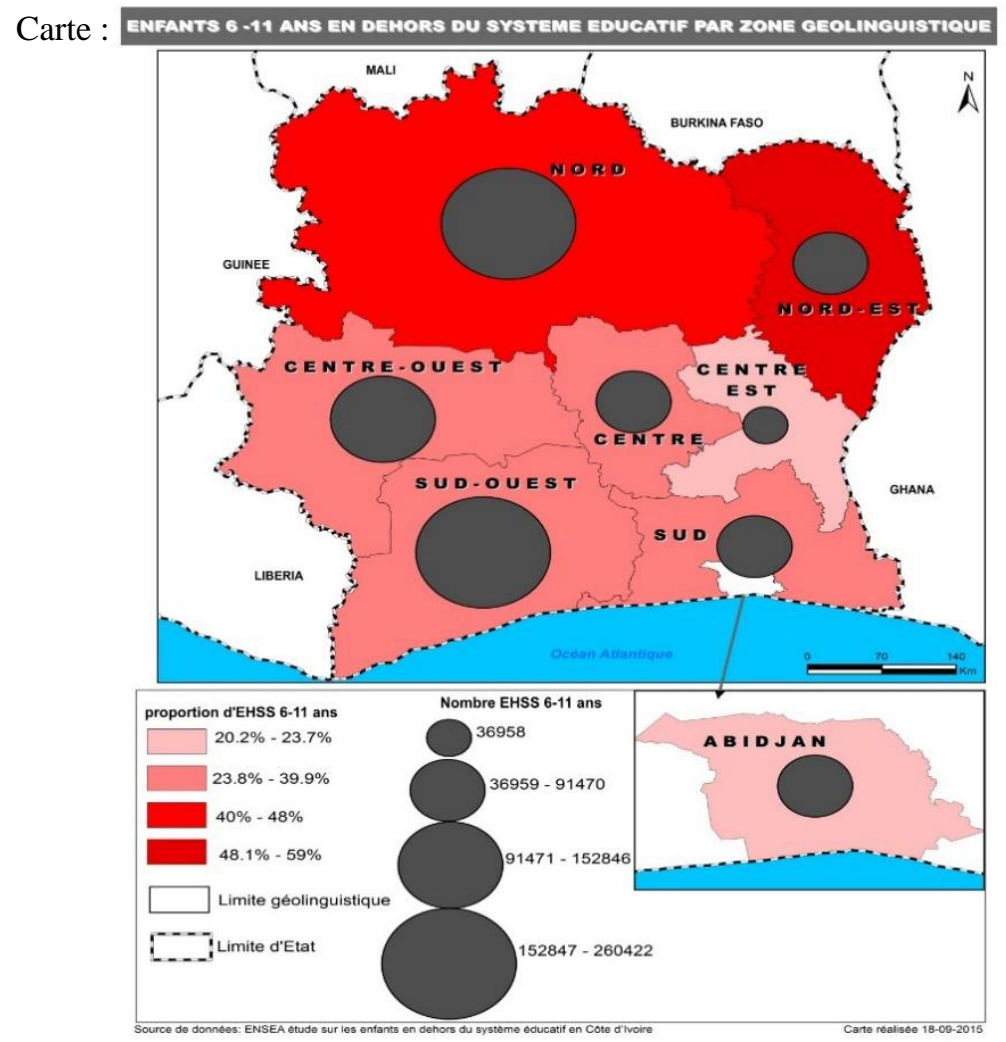

Tableau : Estimations faites selon les cinq dimensions de l'exclusion scolaire

\begin{tabular}{|c|c|c|c|c|c|}
\hline $\begin{array}{c}\text { Nombre } \\
\text { d'enfants exclus }\end{array}$ & 1850951 & 977739 & 797137 & 705823 & 123416 \\
\hline $\begin{array}{l}\text { Dimensions } \\
\text { d'exclusion }\end{array}$ & DE1 & DE2 & DE3 & DE4 & DE5 \\
\hline & $\begin{array}{l}\text { Enfant ayant } \\
\text { l'âge d'aller } \\
\text { au } \\
\text { préscolaire } \\
\text { qui ne sont } \\
\text { pas } \\
\text { scolarisés }\end{array}$ & $\begin{array}{l}\text { Enfants } \\
\text { ayant l'âge } \\
\text { d'aller au } \\
\text { primaire qui } \\
\text { ne sont pas } \\
\text { scolarisés }\end{array}$ & $\begin{array}{l}\text { Enfants } \\
\text { ayant l'âge } \\
\text { d'aller au } \\
\text { secondaire } \\
1 \text { qui ne } \\
\text { sont pas } \\
\text { scolarisés }\end{array}$ & $\begin{array}{l}\text { Elèves du } \\
\text { primaire } \\
\text { qui } \\
\text { risquent } \\
\text { d'abandon } \\
\text { ner }\end{array}$ & $\begin{array}{l}\text { Elèves du } \\
\text { secondaire } 1 \\
\text { qui risquent } \\
\text { d'abandonner }\end{array}$ \\
\hline
\end{tabular}

Le parcours scolaire des enfants et des adolescents en Côte d'Ivoire révèle une grande déperdition des effectifs tout au long de ce parcours. Dans les conditions actuelles d'éducation, sur une cohorte de 100 enfants, près d'un quart $(24,6 \%)$ n'accède jamais à l'école, $19,8 \%$ n'achèvent pas le primaire, $14,5 \%$ n'entrent pas au premier cycle du secondaire, $23,9 \%$ n'achèvent pas 
ce premier cycle. C'est le même constat dans de nombreux pays d'Afrique subsaharienne où près de $20 \%$ des enfants scolarisés dans le primaire n'y resteront probablement pas jusqu'à la dernière classe de l'enseignement primaire. Les niveaux d'abandons restent également très élévés dans cette partie du monde avec 10 millions d'enfants qui abandonnent l'école primaire chaque année (UNESCO, 2015).

Graphique 2 : Le parcours scolaire des enfants en âge d'aller à l'école

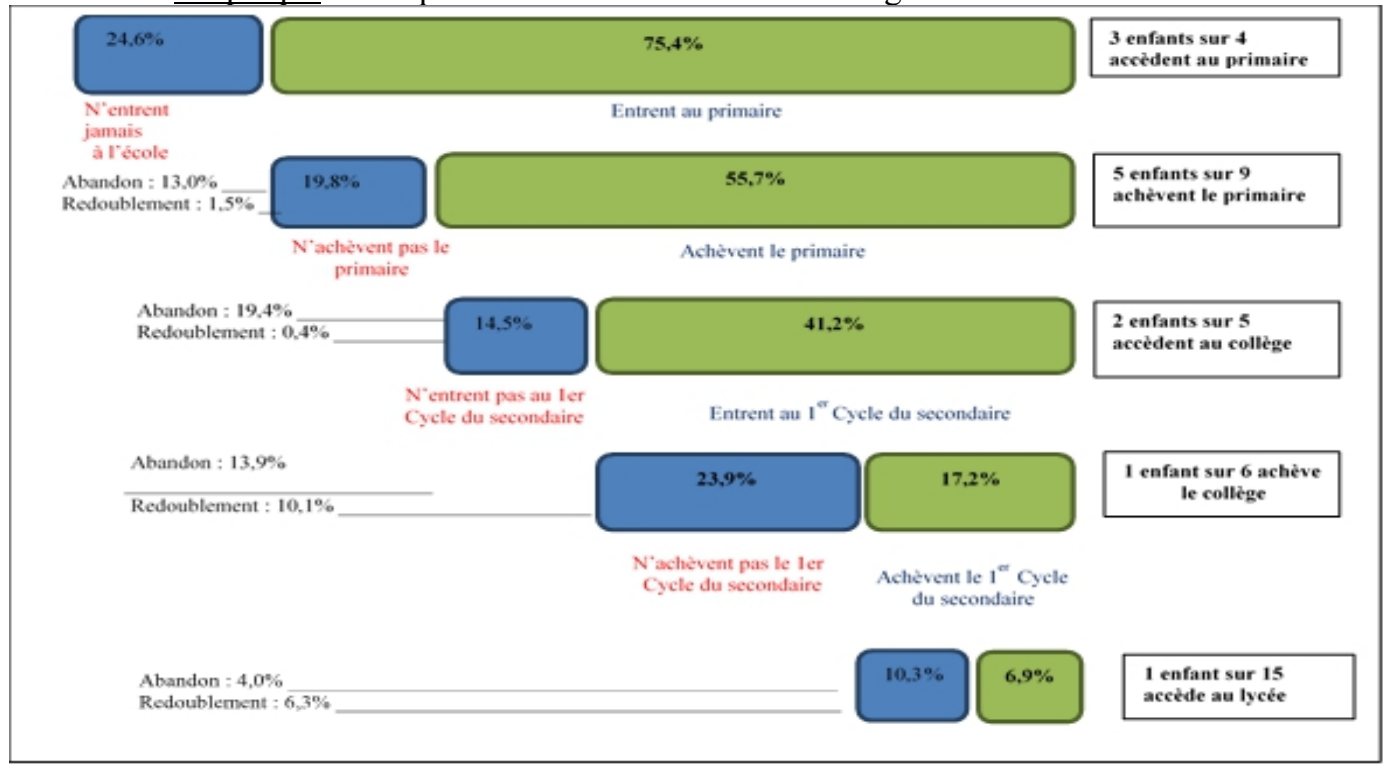

\section{Discussions : l'exclusion scolaire peut-elle impactée les ambitions économiques du pays?}

Depuis sa sortie de la crise politique en 2011, la Côte d'Ivoire est devenue l'une des économies les plus dynamiques d'Afrique, voire du monde. Elle enregistre un taux de croissance record qui devrait se maintenir autour 7,6\% en 2017. Cette bonne performance s'explique par le rebond de l'agriculture aidée par une pluviométrie favorable et des prix des matières premières à la hausse (Groupe Banque Mondiale, 2018). Du coup, le pays s'est fixé comme objectif ambitieux d'atteindre l'émergence économique.

Contrairement aux autres régions du monde, la fécondité, en Afrique subsaharienne, demeure marquée par un niveau globalement plus élevé et un rythme de baisse plus lent. Selon l'UNICEF (2017), les enfants représentent la majorité de la population dans environ un tiers des 55 États membres de l'Union Africaine et leur nombre dépassera le milliard d'ici 2055 sur le continent. Cette croissance démographique soutenue implique des enjeux économiques, sociaux et politiques. L'étude des transitions démographiques et économiques de certains pays d'Asie a pu montrer dès la fin des années 1990 que les changements de la structure par âge peuvent conduire à un avantage 
économique temporaire, qualifié de " dividende démographique » (INED, 2017). En effet, la modification de la pyramide des âges, à travers la baisse de la fécondité, va favoriser une maximisation de la population en âge de travailler par rapport à la population jeune à charge. Ainsi, les pays pourront exploiter une opportunité de croissance économique rapide avec les bons investissements économiques, sociaux et politiques élaborés en matière de santé, d'éducation, de gouvernance et d'économie (AUC et CEA, 2013).

C'est par ce cheminement que la Côte d'Ivoire envisage passer pour atteindre l'émergence. En effet, pour tirer parti de ce dividende démographique, le pays doit investir dans le capital humain afin de créer des opportunités pour le développement d'une main-d'œuvre qualifiée dans tous les secteurs d'activités. En d'autres termes, la population active doit être dotée du savoir-faire et de l'expertise nécessaires pour travailler de manière productive (UNFPA, 2014). L'accès à l'école, la participation et l'achèvement ainsi que la qualité de l'enseignement doivent s'imposer de sorte à avoir des ressources humaines de qualité pour booster le monde du travail en pleine évolution. Par ailleurs, l'éducation devra permettre au pays de réduire le taux de pauvreté, le nombre des personnes à charge dans chaque famille et elle apporte aussi directement les compétences nécessaires pour accroître ses revenus (UNESCO, 2014). Le système éducatif ivoirien doit donc permettre aux jeunes de terminer leur scolarité.

Or, à ce jour, des millions d'enfants et d'adolescents de 3-24 ans sont exclus du système éducatif soit parce qu'ils n'iront jamais à l'école, soit parce qu'ils ont abandonné ou encore risquent de quitter prématurément l'école. Dans un rapport de la Banque Mondiale (2017), il ressort que le taux moyen d'espérance de vie scolaire ${ }^{3}$ en Côte d'Ivoire se situait autour de 8 ans en 2015, alors qu'il atteignait 12 ans au Cap-Vert, autour de 14 ans dans les pays émergents. Ce nombre total d'années d'éducation est faible traduisant ainsi un niveau de scolarisation et d'instruction insuffisant de la population. Ce niveau de scolarisation n'est pas en mesure de favoriser l'atteinte du seuil de capitalisation de l'humain, premier facteur d'une politique de croissance durable (Akindès, 2017). Dans l'optique où la fécondité enclenche une baisse rapide sous l'effet de la hausse de la prévalence contraceptive, le pays ne pourra même pas tirer profit du bonus démographique. Car les jeunes et les adultes seront relativement plus nombreux dans la population active mais ils ne seront pas assez formés pour créer une opportunité de croissance économique. Malgré l'objectif de la scolarisation obligatoire des enfants de 616 ans que s'est fixé la Côte d'Ivoire, la scolarisation universelle est loin d'être atteinte ainsi que l'achèvement universel d'un enseignement de base de dix

${ }^{3}$ L'espérance de vie scolaire (EVS) est définie comme le nombre total d'années d'éducation formelle dont une personne d'un âge donné peut espérer bénéficier (y compris les années de redoublement). 
ans. Investir donc dans l'éducation s'avère comme indispensable au progrès économique.

\section{Conclusion}

La problématique des enfants en dehors de l'école est une question de droit de l'homme que se doivent de résoudre dans l'urgence les gouvernements africains. Si de grands progrès ont été accomplis de par le monde en la matière depuis le début des années 2000, l'Afrique et notamment l'Afrique de l'Ouest et du Centre sont les régions où les progrès ont été les plus lents. La Côte d'Ivoire compte au nombre des pays où de réels progrès sont attendus. En effet, plus de 6 millions d'enfants et d'adolescents étaient exclus de l'école en 2015. Avec quel capital humain, le pays peut espérer atteindre l'émergence économique tant prônée? Car les individus exclus du système scolaire aujourd'hui, seront les exclus de la croissance économique de demain. Les résultats de cette croissance économique seront captés par une élite restreinte ayant bénéficié d'une bonne éducation. Le danger de cette exclusion est réel à court et long terme. Le cas des «microbes », ces bandes de jeunes et d'adolescents en déshérence qui sèment le chaos et la désolation sur leur passage devrait interpeller les autorités. Pour Akindès (2018), le phénomène d'enfants microbes « est un signe avant-coureur de l'apartheid économique et social vers lequel nous nous dirigeons ».

Pour juguler le problème des enfants hors du système éducatif, la Côte d'Ivoire doit prendre des mesures notamment des actions en faveur de la lutte contre la pauvreté des ménages, facteur essentiel à l'origine de la sortie des enfants du système scolaire. Ces mesures sont également relatives aux changements nécessaires des mentalités devant conduire les parents vers une responsabilité accrue vis-à-vis de la scolarisation des jeunes filles et la reconnaissance de l'égalité des chances pour les jeunes garçons et les jeunes filles.

\section{References:}

1. Agepe, (2012). Situation de l'emploi en Côte d'Ivoire en 2012. Enquête emploi auprès des ménages en 2012.

2. Akindès, K. (2018). Les enfants "microbes" sont un signe de l'apartheid économique qui s'installe en Côte d'Ivoire.

3. https://www.lemonde.fr/afrique/article/2018/04/01/les-enfantsmicrobes-sont-un-signe-de-l-apartheid-economique-qui-s-installe-encote-d-ivoire_5279403_3212.html

4. Akindès, K. (2017). Inégalités en Côte d'Ivoire : priorité à l'éducation. https://ideas4development.org/education-cote-divoire/

5. AUC et CEA (2013). L'Afrique et le défi de la réalisation du dividende démographique. $2 \mathrm{p}$. 
6. Fadiga, K. (2006). Efforts et effets de l'adaptation scolaire dans les contextes de crise : analyse avec références particulières à la Côte d'Ivoire, au Libéria et à la Somalie. Mars 2006, 9 p.

7. Groupe Banque Mondiale (2018). Situation économique en Côte d'Ivoire. Aux portes du paradis. Sixième édition, $62 \mathrm{p}$.

8. Groupe Banque Mondiale (2017). Situation économique en Côte d'Ivoire. Le défi des compétences. Quatrième édition, 51p.

9. INED (2017). Dividende démographique et fécondité en Afrique Subsaharienne.

https://www.ined.fr/fr/actualites/rencontresscientifiques/seminairescolloques-ined/dividende-demographique/

10. Mason, A. (2003). Capitalizing On the Demographic Dividend. In: Population and poverty, UNFPA, $\mathrm{N}^{\circ} 8$, pp.39-48.

11. Mingat, A. \& Sosale, S. (2001). Problèmes de politique éducative relatifs au redoublement à l'école primaire dans les pays d'Afrique Sub-saharienne. PSAST/AFTHD, Banque Mondiale.

12. Ministère d'Etat, Ministère du Plan et du Développement (2006). Rapport national sur l'état et le devenir de la population de la Côte d'Ivoire. Abidjan, 190p.

13. RESEN (Rapport d'État du Système Éducatif National). 2016. Rapport d'Etat sur le système éducatif national pour une politique éducative plus inclusive et plus efficace. Washington, 320p.

14. UNFPA (2014). Le dividende démographique peut accélérer la croissance économique et favoriser le développement national. $8 \mathrm{p}$.

15. UNICEF (2017). Génération 2030 : rapport sur la croissance démographique en Afrique. https://www.unicef.fr/contenu/espacemedias/generation-2030-rapport-sur-la-croissance-demographique-enafrique

16. UNESCO (2016). Leaving no, one behind: How far on the way to universal primary and secondary education? policy paper 27/fact sheet 37? $16 \mathrm{p}$.

17. UNESCO (2015). Faits et chiffres : Progrès et défis de l'éducation en Afrique subsaharienne.

18. http://www.unesco.org/new/fr/education/themes/leading-theinternational-agenda/education-for-all/single-

view/news/facts_and_figures_sub_saharan_africas_education_progres s/

19. UNESCO (2014). Le développement durable commence par l'éducation. Comment l'éducation peut contribuer à la réalisation des objectifs proposés pour l'après 2015. France. 20p. 